\title{
Fault Detection of a Reusable Rocket Engine using Phase Plane Trajectory of Feature Vectors
}

\author{
Seiji Tsutsumi ${ }^{1}$, Miki Hirabayashi ${ }^{2}$, Daiwa Sato ${ }^{3}$, Masaharu $\mathrm{Abe}^{4}$, Kaname Kawatsu ${ }^{5}$, Masaki Sato $^{6}$, Toshiya Kimura ${ }^{7}$, and \\ Tomoyuki Hashimoto ${ }^{8}$ \\ ${ }^{1,2,3}$ Research Unit III, Research and Development Directorate, Japan Aerospace Exploration Agency (JAXA), Sagamihara, \\ Kanagawa, 252-5210, Japan \\ tsutsumi.seiji@jaxa.jp \\ hirabayashi.miki@jaxa.jp \\ satoh.daiwa@jaxa.jp \\ ${ }^{4}$ Aerospace Engineering Solution Division, Business Headquarters, Ryoyu Systems Co., Ltd., Nagoya, Aichi, 455-0024, \\ Japan \\ abe.masaharu@jaxa.jp \\ ${ }^{5}$ Research Unit III, Research and Development Directorate, Japan Aerospace Exploration Agency (JAXA), Tsukuba, Ibaraki, \\ 305-8505, Japan \\ kawatsu.kaname@jaxa.jp \\ 6,7,8 Research Unit IV, Research and Development Directorate, Japan Aerospace Exploration Agency (JAXA), Kakuda, \\ Miyagi, 981-1525, Japan \\ sato.masaki@jaxa.jp \\ kimura.toshiya@jaxa.jp \\ hashimoto.tomoyuki@jaxa.jp
}

\begin{abstract}
A bivariate time-series analysis based on the phase plane trajectory of feature vectors extracted by principal component analysis is developed for fault detection in a reusable liquid-propellant rocket engine. Static-firing test results of the reusable rocket engine obtained at the Japan Aerospace Exploration Agency are employed for demonstration of the present method. The present method successfully detected temperature sensor failure from 19 firing tests of 62 sensors, even in the deviation of the engine operational sequence between the static-firing tests. The present method was also able to detect the system failure from 23 firing tests. Furthermore, the ability to distinguish the system and sensor failure was demonstrated.
\end{abstract}

\section{INTRODUCTION}

Fault detection and diagnostics is applied in industrial systems. Regarding a rocket engine, a study of health

Seiji Tsutsumi et al. This is an open-access article distributed under the terms of the Creative Commons Attribution 3.0 United States License, which permits unrestricted use, distribution, and reproduction in any medium, provided the original author and source are credited. monitoring was conducted for the Space Shuttle Main Engine (SSME), which was the first reusable rocket engine put to practical use (Hawman et al., 1990). However, disassembly inspection and reassembly of the SSME were well-known to be required after every flight, which increased maintenance costs and duration of the maintenance period. As with aircraft engines, minimizing maintenance for the next flight, such as eliminating disassembly inspection, is essential for reusable rocket engines. For that purpose, a technique to evaluate remaining lifetime, non-destructive inspection technology, and a fault detection and diagnostics technique are required. Within these three requirements, this study focuses on the development of fault detection and diagnostics.

Studies on the fault detection and diagnostics of liquidpropellant rocket engines conducted mainly during the 1990s were surveyed in the reference. (Wu, 2005). Recently, research and development of fault detection for liquidpropellant rocket engines were also conducted (Schwabacher et al., 2009, Iannetti et al., 2015, Sato et al., 2017). Before entering a discussion of the methodology, this study initially surveyed the requirements for the fault detection and diagnostics of liquid-propellant rocket engines, as summarized in Section 2. To the extent of the authors' investigation, there is no well-established methodology to 
cope with those requirements. Therefore, a bivariate timeseries analysis is developed for fault detection, based on principal component analysis (PCA). PCA is often used as a multivariate method of fault detection (Jolliffe, 2002). In the present method, PCA is applied to each sensor data to extract a feature vector, and a phase plane trajectory of the feature vectors for two sensors is generated. Then, the difference of the phase plane trajectories between engine firing tests are evaluated as a scalar value (called the degree of dissimilarity), and a conventional univariate fault detection method is employed to find faults in the firing test. The proposed method does not target on-board judgment to prevent destructive events during flight, but is intended for use as a ground system after landing to reduce maintenance activities for the next flight. Research and development of a reusable sounding rocket (RSR) is being conducted at the Japan Aerospace Exploration Agency (JAXA) (Inatani et al., 2010, Nonaka et al., 2015). As part of this research, static-firing tests of a reusable liquid-propellant rocket engine for the RSR (called RSR engine) were conducted. (Kimura et al., 2016) This study employs those firing test results for demonstration of the proposed method.

\section{REQUIREMENTS FOR FAULT DETECTION AND DIAGNOSTICS}

This study initially investigated the requirements for fault detection and diagnostics of liquid-propellant rocket engines, and thus identified eight items. These items should be kept in mind for the research and development of fault detection and diagnostics of liquid-propellant rocket engines.

1. Training data available for the fault detection and diagnostics are limited. In particular, failure data are extremely limited.

2. Sensors installed in the engine occasionally fail. A fault detection methodology is thus required to distinguish between sensor and system failure.

3. There are individual differences in the liquid-propellant rocket engine. And, even in the same engine, the operating conditions occasionally drift during firing.

4. The operating conditions of the liquid-propellant rocket engine change dynamically. The steady-state condition as well as such transient conditions as startup, shutdown, and thrust control must be taken into account.

5. The number of sensors and the arrangement thereof are not always appropriate for fault detection and diagnostics. Thus, optimization of the sensor arrangement may be important.

6. Due to the restrictions on sensor installation in the engine, there may be a gap between measured values and physics.

7. The study of fault diagnostics methodologies and practical examples are limited.
8. Considering the model-based approach, validation data for the simulation model including failure are limited.

\section{RSR ENGINE}

The RSR engine was developed to demonstrate the capability of wide-range throttling, accurate control of operational sequence, and on-board health monitoring. Another purpose of the RSR engine was to verify long-life durability that could withstand over 100 flights. Specifications of the RSR engine are listed in Table 1. The RSR engine employs liquid hydrogen $\left(\mathrm{LH}_{2}\right)$ and liquid oxygen $(\mathrm{LOX})$ as the fuel and oxidizer, respectively, and generates $40 \mathrm{kN}$ of thrust at $100 \%$ throttling at sea level. The expander bleed cycle is employed. Schematic of the RSR engine is shown in Fig. 1. In the expander bleed cycle, a fuel turbopump (FTP) and an oxidizer turbopump (OTP) are driven by hydrogen heated by cooling the combustion chamber and nozzle wall. Considering the reusability of the engine, chamber pressure is set to $3.4 \mathrm{MPa}$, and specific impulse of 320 seconds is achieved at $100 \%$ thrust. As this engine is designed for a launch vehicle capable of vertical take-off and landing, reignition and throttling from $40 \%$ to $100 \%$ are available to change the trajectory and decelerate for returning to the ground. A total of 54 firings was conducted in the static-firing test of the RSR engine, and the engine was ignited 142 times. More than 350 sensors were installed in both the RSR engine and the ground facility. Details of the RSR engine and the static-firing test are given in the references (Sato et al., 2014, Kimura et al., 2016).

Table 1. Specifications of the RSR engine.

\begin{tabular}{|l|c|}
\hline Propellant & $\mathrm{LH}_{2} / \mathrm{LOX}$ \\
\hline Engine cycle & Expander bleed \\
\hline Throttling range, \% & $40-100$ \\
\hline Thrust (sea level), $\mathrm{kN}$ & 40 \\
\hline Specific impulse, sec & 320 \\
\hline Chamber pressure, $\mathrm{MPa}$ & 3.4 \\
\hline Mixture ratio & 6.0 \\
\hline
\end{tabular}

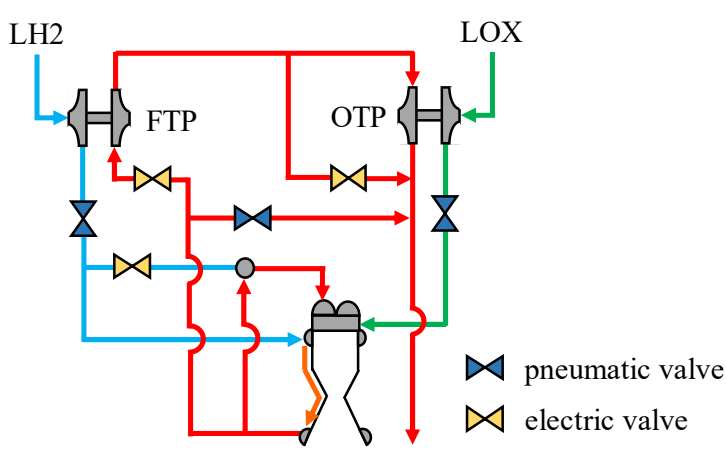

Figure 1. Schematic of the RSR engine. 


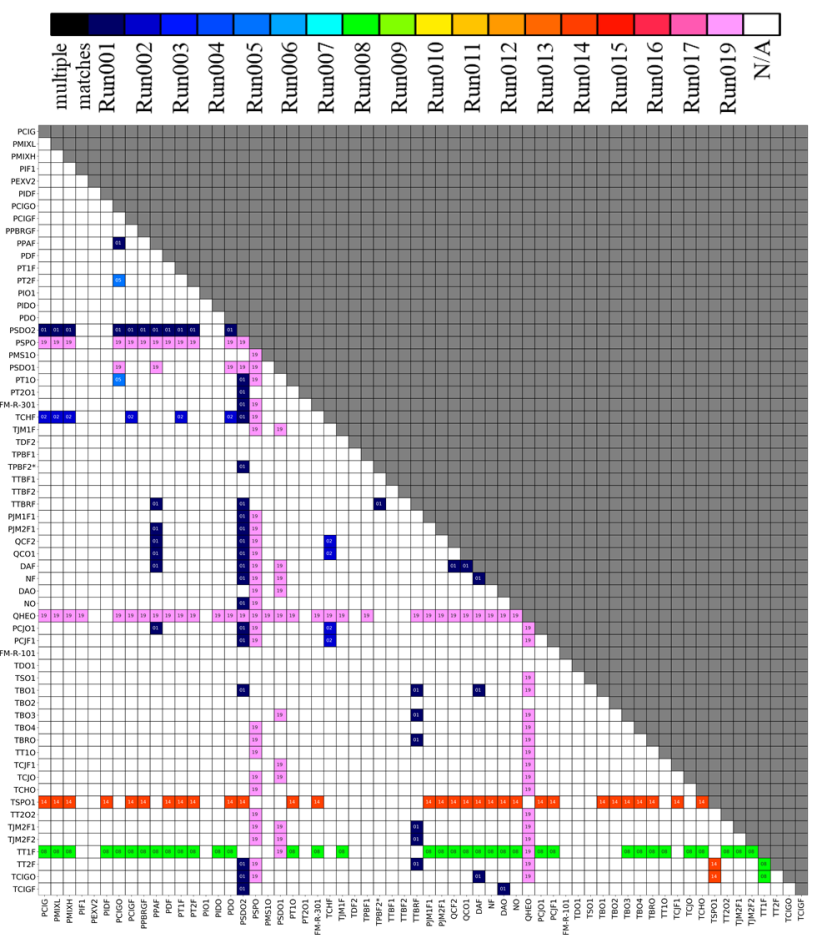

(a) $99 \%$.

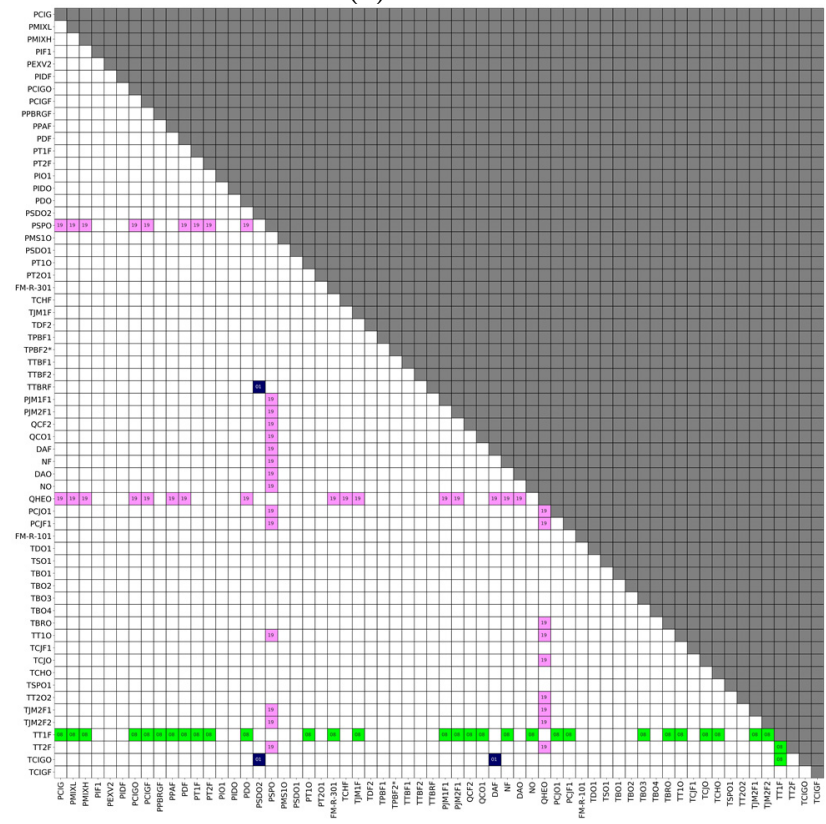

(b) $99.5 \%$.

Figure2. SEM of $1^{\text {st }}$ PCA mode for TT1F sensor failure detection.

\section{BIVARIATE TIME-SERIES ANALYSIS BASED ON PHASE PLANE TRAJECTORY OF FEATURE VECTORS}

This study aims to develop a fault detection technique for the liquid-propellant rocket engine in a reusable launch vehicle after landing to reduce maintenance activities for the next flight. Considering the technical challenges listed in Section
2 , univariate time-series analysis is not an option because it is impossible to identify sensor and system failure. Therefore, bivariate time-series analysis that examines the relationship between two sensors is employed.

Let $x_{t}^{s_{i}}$ be the measured value at time $t(t=1,2, \cdots, T)$ of the $i^{\text {th }}$ sensor $(i=1,2, \cdots, S)$. Time-series data measured by sensor $s_{i}$ is represented by $\mathcal{D}^{s_{i}}=\left[x_{1}^{s_{i}}, x_{2}^{s_{i}}, \cdots, x_{T}^{s_{i}}\right]^{\top}$. Here, $\mathrm{T}$ denotes transposition. Standardized time-series data $\left(\widetilde{\mathcal{D}}^{s_{i}}\right)$, is evaluated based on the mean and variance of a normal firing test. PCA is then applied to $\widetilde{\mathcal{D}}^{s_{i}}$ to extract the features (modes) of $\widetilde{\mathcal{D}}^{s_{i}}$. System and sensor noise are potentially included in the measured value of the sensor. Depending on the fault detection technique proposed so far, it is important to remove noise in advance. While extracting features (modes) using PCA entail the removal of unnecessary fluctuations, it is equivalent to a noise filter. In order to apply PCA to univariate time-series data $\left(\widetilde{\mathcal{D}}^{s_{i}}\right)$, a sliding window having the width of $W$ is applied for extraction, according to Fujimaki et al. (2005). Let the set of time-series data extracted by the sliding window at $t$ be $\widetilde{\mathcal{D}}_{t}^{s_{i}}=\left[x_{t}^{s_{i}}, x_{t+1}^{s_{i}}, \cdots, x_{t+W-1}^{s_{i}}\right]^{\top}$, then a matrix $\widetilde{\mathcal{D}}_{\text {win }}^{s_{i}}=$ $\left[\widetilde{\mathcal{D}}_{1}^{s}, \widetilde{\mathcal{D}}_{2}^{s}, \cdots, \widetilde{\mathcal{D}}_{T-W+1}^{s}\right]^{\top}$ with a size of $(T-W+1) \times W$ is obtained. PCA is applied to matrix $\widetilde{\mathcal{D}}_{\text {win }}^{s_{i}}$, and principal component score $\boldsymbol{Y}^{s_{i}(m)}=\left[y_{1}^{s_{i}(m)}, y_{2}^{s_{i}(m)}, \cdots, y_{T-W+1}^{s_{i}}{ }^{(m)}\right]^{\top}$ of the $m^{\text {th }}$ mode is computed. In practice, sensor data will appear on a non-linear hyperplane, and the kernel PCA is essential to extract the proper feature vectors. However, selection of the kernel function depends on the characteristics of each sensor data, and it is almost impossible to find the best kernel one by one. Therefore, the kernel PCA is not employed in this study.

The $m^{\text {th }}$ principle component scores of sensors $s_{i}$ and $s_{j}$ are plotted on the horizontal and vertical axes, respectively, and a two-dimensional phase plane trajectory with time $t$ as a parameter $\quad \mathcal{O}^{(m)}\left(s_{i}, s_{j}\right)=\left[\boldsymbol{Y}^{s_{i}(m)}, \boldsymbol{Y}^{s_{j}(m)}\right]=$ $\left[\left[y_{1}^{s_{i}(m)}, y_{1}^{s_{j}(m)}\right],\left[y_{2}^{s_{i}(m)}, y_{2}^{s_{j}(m)}\right], \cdots\left[y_{T-W+1}^{s_{i}}{ }^{(m)}, y_{T-W+1}^{s_{j}}{ }^{(m)}\right]\right]^{\top}$ is obtained. The phase plane trajectory $\mathcal{O}^{(m)}\left(s_{i}, s_{j}\right)$ shows the relationship between sensors of $s_{i}$ and $s_{j}$. Comparing the shape of the phase plane trajectory between firing tests, it is possible to find a firing test with an existing fault.

To compare the shape of the phase plane trajectory $\mathcal{O}^{(m)}\left(s_{i}, s_{j}\right)$ between firing tests, the difference in shape is quantified as a degree of dissimilarity. There are several methods to quantitatively evaluate the shape difference, such as a complex autoregressive model (Kurita et al., 1994), dynamic time warping (DTW) (Bahlmann and Burkhardt, 2004), and a convolution neural network. In this study, DTW is employed. DTW is used for the recognition of speech and handwriting due to its ability to perform pattern matching 
even for different waveforms by people. As described in Section 2, the timing of the engine operational sequence varies between flights or static-firing tests due to control input. Individual differences of the engine and drift of the operating conditions in the same engine also induce variation of this timing. DTW is expected to evaluate the degree of dissimilarity between firing tests with robustness, even in those situations. $\mathcal{O}_{R_{0}}^{(m)}\left(s_{i}, s_{j}\right)$ and $\mathcal{O}_{R_{k}}^{(m)}\left(s_{i}, s_{j}\right)$ denote the phase plane trajectories consisting of the $m^{\text {th }}$ mode of sensors $s_{i}$ and $s_{j}$ for the two firing tests of $R_{0}$ and $R_{k}$, respectively. $R_{0}$ is set to a normal firing test, and $R_{k}$ denotes the one of the $K$ firing tests $(k=1,2, \cdots, K)$. Local distance between $\mathcal{O}_{R_{0}}^{(m)}\left(s_{i}, s_{j}\right)$ at $t$ and $\mathcal{O}_{R_{k}}^{(m)}\left(s_{i}, s_{j}\right)$ at $t^{\prime}$, expressed as $g_{s_{i}, s_{j}}^{(m)}\left(t, t^{\prime}\right)$, is evaluated with the Euclidean distance as follows:

$$
\begin{aligned}
& g_{s_{i}, s_{j}}^{(m)}\left(t, t^{\prime}\right) \\
& =\left\|\left[y_{t}^{s_{i}(m)}, y_{t}^{s_{j}(m)}\right]^{\left(R_{0}\right)}-\left[y_{t^{\prime}}^{s_{i}(m)}, y_{t^{\prime}}^{s_{j}(m)}\right]^{\left(R_{k}\right)}\right\|_{2}
\end{aligned}
$$

Then the DTW distance between $\mathcal{O}_{R_{0}}^{(m)}\left(s_{i}, s_{j}\right)$ and $\mathcal{O}_{R_{k}}^{(m)}\left(s_{i}, s_{j}\right), G_{s_{i}, s_{j}}^{(m)}\left(R_{0}, R_{k}\right)$, is obtained. Note that $\mathcal{O}_{R_{k}}^{(m)}\left(s_{i}, s_{j}\right)$ is resampled by the homogenous points of 4096 to standardize the length of the operational time.

After evaluating the DTW distance for all the firing tests denoted by $\boldsymbol{G}=$ $\left\{G_{s_{i}, S_{j}}^{(m)}\left(R_{0}, R_{1}\right), G_{s_{i}, s_{j}}^{(m)}\left(R_{0}, R_{2}\right), \cdots, G_{s_{i}, s_{j}}^{(m)}\left(R_{0}, R_{K}\right)\right\}$, univariate methods for fault detection can be employed. In this study, $\boldsymbol{G}$ assumes to follow the Gaussian distribution $\mathcal{N}$, and anomaly score $\alpha$ of $R^{\prime}$ is defined as the negative log-likelihood:

$$
\begin{aligned}
& \alpha\left(G_{s_{i}, s_{j}}^{(m)}\left(R_{0}, R^{\prime}\right)\right) \\
& \equiv-\frac{K-1}{K+1} \ln \left\{\mathcal{N}\left(G_{s_{i}, s_{j}}^{(m)}\left(R_{0}, R^{\prime}\right) \mid \boldsymbol{G}\right)\right\}
\end{aligned}
$$

As a result, the distribution of anomaly score $\alpha$ follows the F-distribution with parameters 1 and $K-1$ under Hotelling's $\mathrm{T}^{2}$ theory.

$$
\alpha\left(G_{s_{i}, s_{j}}^{(m)}\left(R_{0}, R^{\prime}\right)\right) \sim \mathcal{F}(1, K-1)
$$

If $K \gg 1$, anomaly score $\alpha$ follows the chi-square distribution with a degree of freedom of 1 and scale factor of 1. However, Eq. (3) is employed in this study due to an insufficient number of firing tests $K$.

The present bivariate time-series analysis is applied to the all sensor combinations, the number of which is ${ }_{s} C_{2}$, and then the fault detection results are visualized as a sensor-state estimation matrix (SEM). Figure 2 shows an example of the SEM for the RSR engine that will be discussed later in Section 5.1. The rows and columns represent $S$ sensors. If failure is detected for the combination of $s_{i}$ and $s_{j}$, the element corresponding to these two sensors shows a failure signal. In the case of the RSR engine, the firing test number in which the failure occurred is indicated with color. Note that the diagonal and upper triangular elements are grayed out because the SEM is symmetric. If sensor failure occurred at $s_{i}$, all combinations associated with $s_{i}$ show the failure signal. In the SEM, those signals will appear in a swath element. And in case of system failure, more than one sensor shows failure, and multiple relationships associated with those sensors are broken. Therefore, the failure signal will appear here and there in the SEM. As a result, the appearance of failure signal in the SEM will enable sensor and system failure to be distinguished.

Hyperparameters appearing in this method are the size of the sliding window $(W)$, and the mode $(m)$ of PCA. The $1^{\text {st }}$ mode of PCA corresponds to a dominant feature of the data, and usually has a low frequency component. The $2^{\text {nd }}$ PCA mode corresponds to an additional feature, and usually shows high frequency fluctuation. Higher order modes than the $2^{\text {nd }}$ mode also appear in PCA analysis, however, in this study, the $1^{\text {st }}$ and $2^{\text {nd }}$ PCA modes are only employed for generating the phase plane trajectory. The boundary between the $1^{\text {st }}$ and $2^{\text {nd }}$ PCA modes depends on the size of sliding window $W$. Through the preliminary study, $W$ is fixed to 5 in the following discussion.

Computation of the DTW distance is a hot spot, as its order of computational cost is $O\left(N^{2}\right)$. Therefore, the current method is mainly developed using Python 3, and only DTW that is a hot spot is functionalized using Fortran90. And then, the hyperplane ordering method (Fujino et al., 1991) is applied to DTW for speed-up.

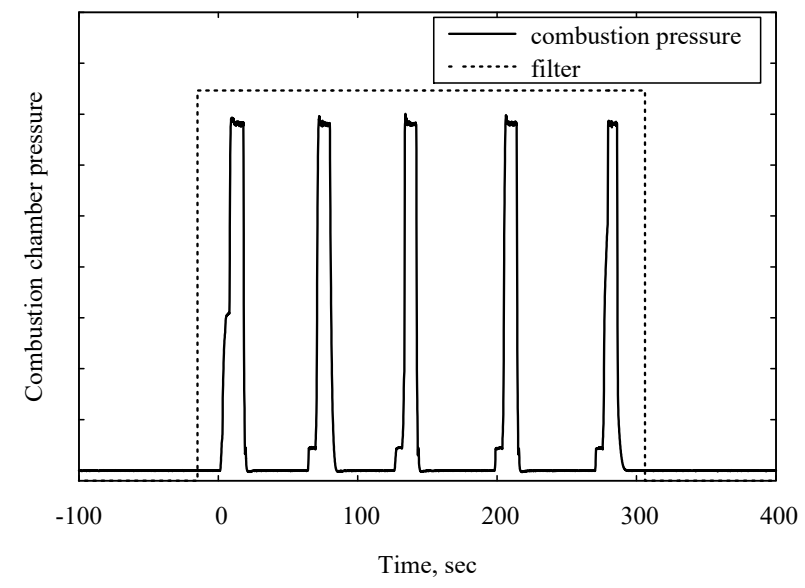

Figure 3. Time history of combustion pressure.

\section{DEMONSTRATION USING RSR ENGINE TESTS}

Although a total of 350 sensors were used in the static-firing test, 62 sensors installed in the RSR engine are chosen in this 
study for demonstration of the present bivariate time-series analysis. The sampling rate of all those sensors was $100 \mathrm{~Hz}$. As mentioned before, a total of 54 firings was conducted in the static-firing test of the RSR engine. The present method is applied to sensor failure that occurred in one of the 54 static-firing tests. The present method is also applied to the system failure intentionally generated for demonstration of the on-board health monitoring system (Kimura et al., 2016). Through that demonstration, the capability of the present method will be discussed.

\subsection{Sensor Failure}

During the static-firing tests, an abnormal event occurred at one of the sheathed thermocouples installed at the inlet line of the FTP turbine, represented as TT1F. During engine operation, the sheath was vibrated by the fluid force of the turbine driving gas due to failure of the sheathed thermocouple, and then noise related to the Karman vortex shedding from the sheath appeared in the measured value. Figure 3 shows the engine operational sequence at the occurrence of this event. In this firing test, the RSR engine was ignited at 0 seconds, and initially started up at $40 \%$ thrust. 8 seconds after ignition, the thrust increased to $100 \%$ for a duration of 10 seconds. Then the RSR engine was stopped. After that, the RSR engine was repeatedly started up four times at $100 \%$ thrust at intervals of roughly 40 seconds. In the static-firing test of the RSR engine, 19 tests were conducted with the same operational sequence. These tests are represented as Run001 to Run019 here. The abnormal event of TT1F occurred in Run008. Figure 4 shows a comparison of abnormal data of TT1F at Run008 with typical normal data at Run009. The result of Run008 only showed fluctuation when the engine was operated at $100 \%$ thrust due to failure of the sheathed thermocouple. It is also observed in Fig. 4 that the timing at which the thrust after reignition reaches $100 \%$ deviates depending on the firing test. As 62 sensors installed in the RSR engine are used here, there are 1,891 combinations of the 62 sensors $\left({ }_{62} C_{2}\right)$. In each combination, the degree of dissimilarity of the phase trajectory is evaluated for 19 firing tests. The measured results of the 62 sensors sampled at $100 \mathrm{~Hz}$ are extracted between the time range shown by the dotted line in Fig. 3, and then analyzed without any preprocessing, such as noise filtering. It took about 50 minutes to analyze the 1,891 combinations of sensors for the 19 firing tests by using 10 cores of Intel Xeon E5-2643. Figures 2 and 5 show the SEMs of the $1^{\text {st }}$ and $2^{\text {nd }}$ PCA modes, respectively, with the threshold for anomaly scores of $99 \%$ and $99.5 \%$. The nominal test case $\left(R_{0}\right)$ is set to Run018 for computing the DTW distance here. The 62 sensors are listed in the rows and columns, and the firing test number detected as a fault is identified as color in each element. In case multiple tests are detected as failure, the element is colored black. Otherwise, the element is colored white. Because the noise that appeared in TT1F as shown in Fig. 4 is related to the Karman vortex, the noise appeared in both the $1^{\text {st }}$ and $2^{\text {nd }}$ PCA modes with a window size $(W)$ of 5 . The failure signals indicating Run008 successfully appear at the swath elements related to TT1F in both the $1^{\text {st }}$ and $2^{\text {nd }}$ PCA mode results. Table 2 summarizes the true positive rate (TPR), true negative rate (TNR), and Fscore. Note that a higher F-score was obtained in the results of the $2^{\text {nd }}$ PCA mode: 0.93 and 0.95 for the $99 \%$ and $99.5 \%$ thresholds, respectively.

The combination of the TT1F and FTP turbine outlet temperature, represented as TT2F, is picked up for detailed discussion. Figure 4 also shows the time-series results of TT2F for Run008 and Run009. The results show a normal profile. Figure 6 shows a comparison of the phase plane trajectories for the $2^{\text {nd }}$ PCA mode of TT1F and TT2F, $\mathcal{O}^{(2)}$ (TT1F, TT2F), for the 19 firing tests. The phase plane trajectory is colored by the time from engine ignition. The difference in shape between the normal and fault tests is obvious. The normal firing tests have a shape like a vertical bar, whereas horizontal bars appeared in Run008. The hierarchical clustering is applied to the degree of dissimilarity between the phase plane trajectories of the 19 firing tests, $G_{\mathrm{TT} 1 \mathrm{~F}, \mathrm{TT} 2 \mathrm{~F}}^{(2)}\left(\mathrm{Run} 018, R_{k}\right)(k=1, \cdots, 17,19)$, with the results shown in Fig. 7. Note that Run008 largely deviates from the other firing tests closely located together. Therefore, a fault is considered to occur in Run008.

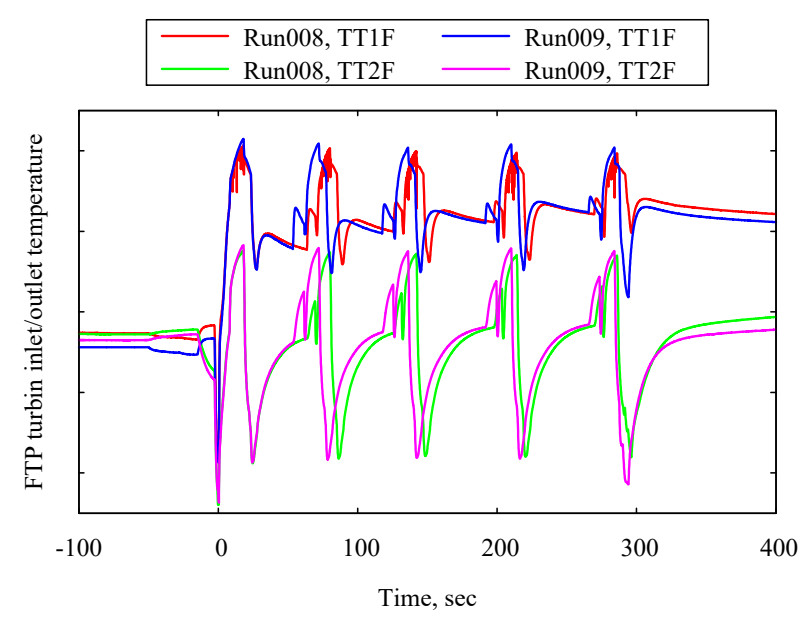

Figure4. Comparison of FTP turbine inlet and outlet

temperature profiles of normal and abnormal firing tests.

Table 2. Prediction results for TT1F sensor failure detection.

\begin{tabular}{|c|c|c|c|c|}
\hline & $\begin{array}{c}1^{\text {st }} \text { PCA } \\
\text { mode, } \\
99 \%\end{array}$ & $\begin{array}{c}1^{\text {st }} \text { PCA } \\
\text { mode, } \\
99.5 \%\end{array}$ & $\begin{array}{c}2^{\text {nd }} \text { PCA } \\
\text { mode, } \\
99 \%\end{array}$ & $\begin{array}{c}2^{\text {nd }} \text { PCA } \\
\text { mode, } \\
99.5 \%\end{array}$ \\
\hline TPR & $89.5 \%$ & $97.2 \%$ & $92.0 \%$ & $98.0 \%$ \\
\hline TNP & $60.7 \%$ & $50.8 \%$ & $93.4 \%$ & $91.8 \%$ \\
\hline F-score & 0.72 & 0.67 & 0.93 & 0.95 \\
\hline
\end{tabular}




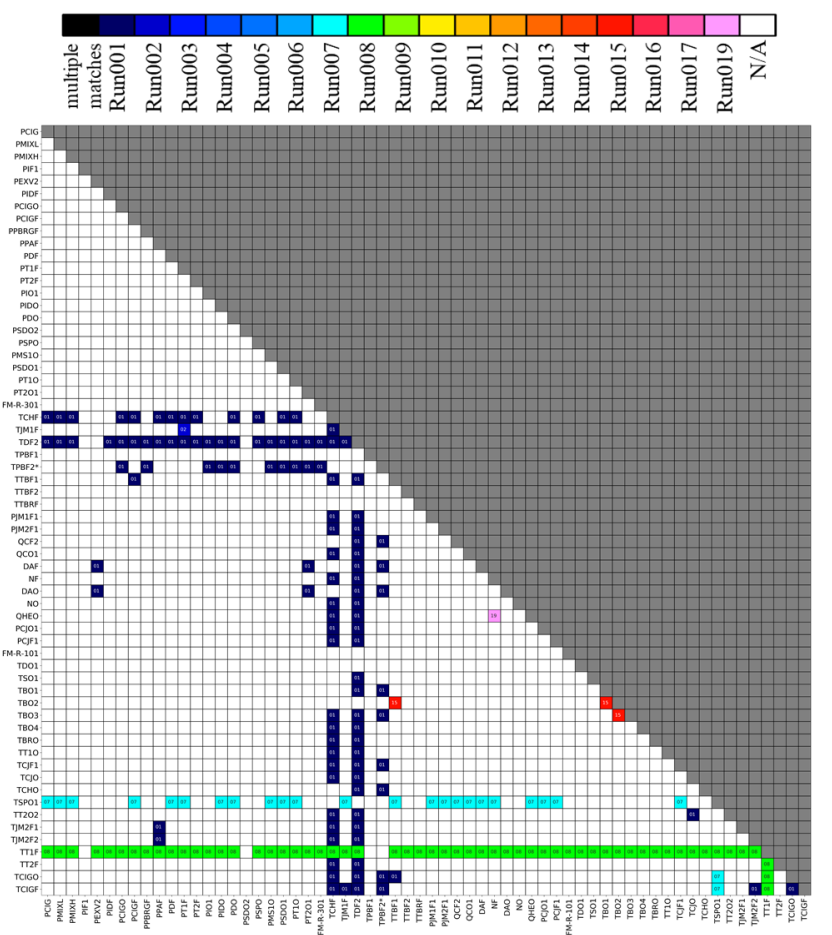

(a) $99 \%$.

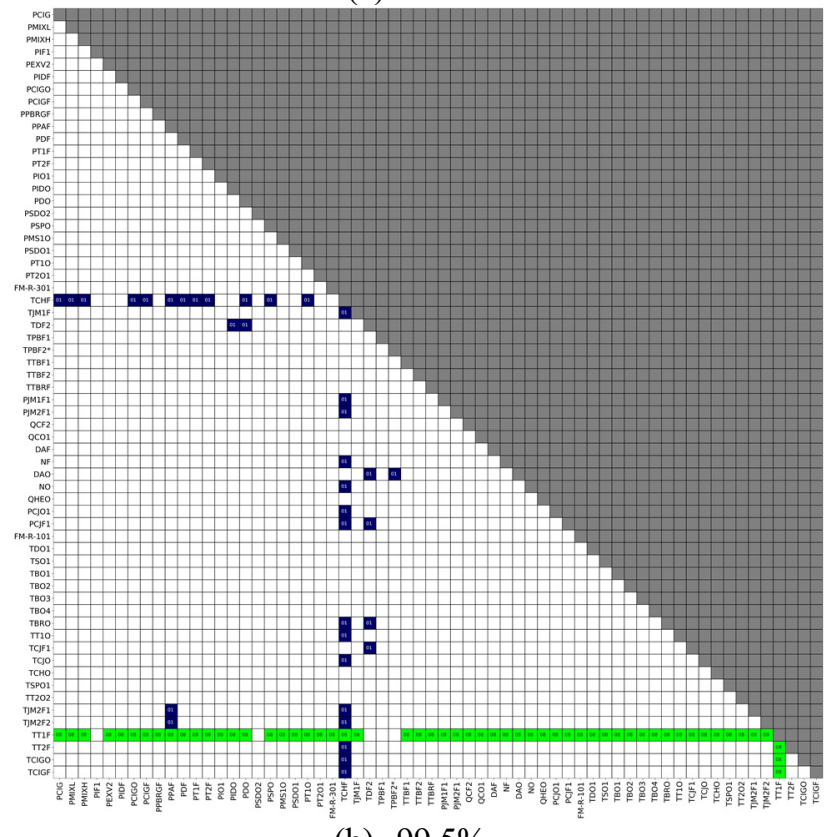

(b) $99.5 \%$.

Figure5. SEM of $2^{\text {nd }}$ PCA mode for TT1F sensor failure

detection.

\subsection{System Failure}

In the series of static-firing tests, the inlet pressure of the FTP inducer was intentionally decreased to demonstrate the onboard health monitoring system. The failure simulated here is one of the major system failures leading to a catastrophe, such as turbopump failure due to overspin. Figure 8 compares the
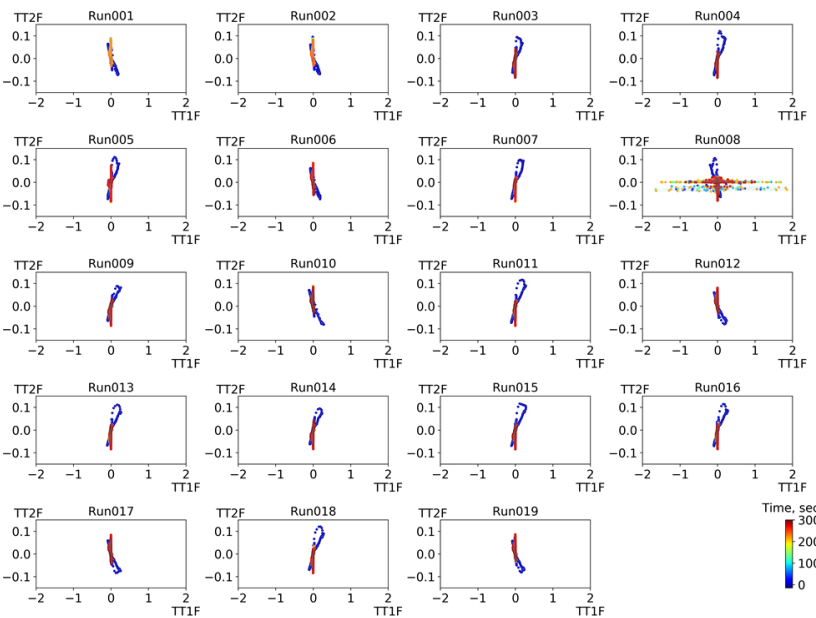

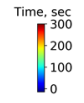

Figure6. Phase plane trajectories $\left(\mathcal{O}_{R_{k}}^{(2)}(\mathrm{TT} 1 \mathrm{~F}, \mathrm{TT} 2 \mathrm{~F})\right)$ for 19 firing tests $(k=1, \cdots, 19)$.

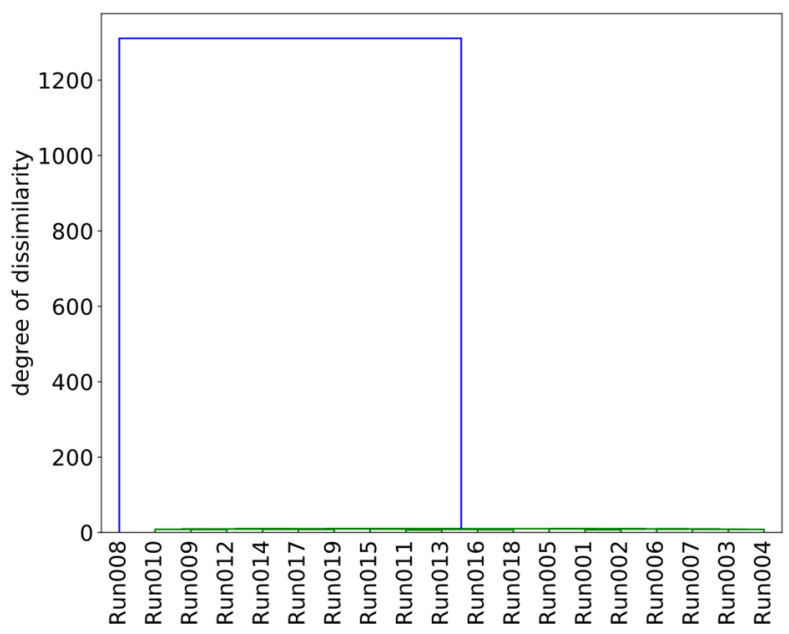

Figure7. Dendrogram of the degree of dissimilarity,

$$
G_{\mathrm{TT} 1 \mathrm{~F}, \mathrm{TT} 2 \mathrm{~F}}^{(2)}\left(\operatorname{Run} 018, R_{k}\right)(k=1, \cdots, 17,19) .
$$

inlet pressure of the FTP inducer, represented as PIF1, between abnormal test (Run022) and normal test (Run023). In the normal test of Run023, PIF shows constant value during $100 \%$ thrust from 8 to $43 \mathrm{sec}$. While, in the abnormal test of Run022, a fuel tank containing $\mathrm{LH}_{2}$ was intentionally blown down to decrease PIF from $18 \mathrm{sec}$. (Kimura et al., 2016).

The present bivariate time-series analysis is also applied to that test as a demonstration of the system failure. In this demonstration, 23 firing tests including the first engine ignition from 0 to 30 seconds of Run001 to Run019 are employed. In the additional firing tests of Run020 to Run023, the thrust started up to $40 \%$ before going to $100 \%$ thrust, same with Run001 to Run019. As mentioned above, Run022 corresponds to the test case with failure. Note that the sensor failure of TT1F at Run008 is also included here. The chamber 


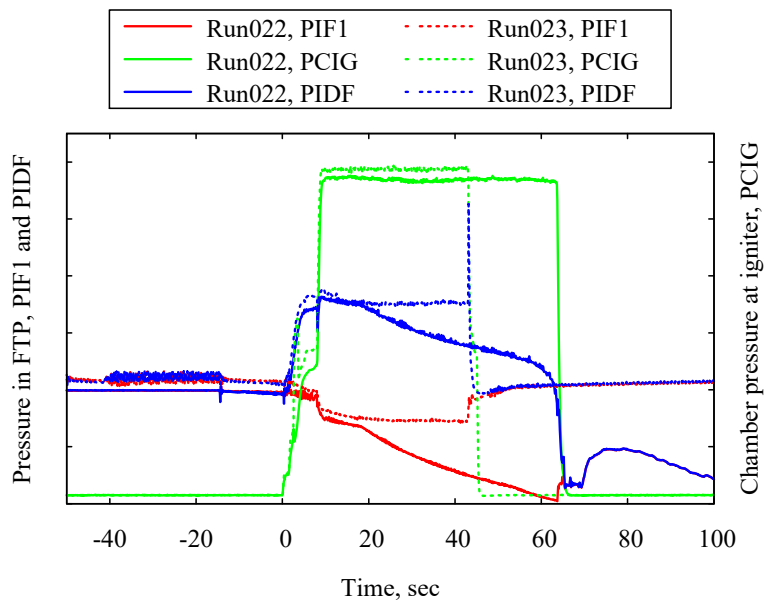

Figure8. Comparison of pressure in FTP inlet and combustion chamber of normal and abnormal firing tests.

pressure measured at igniter, represented as PCIG, is also shown in Fig. 8. According to PCIG, duration of the operation at $100 \%$ thrust is different between Run022 and Run023. The RSR engine stops at 64 seconds for Run022, and 43 seconds for Run023, respectively. The results of PCIG are almost consistent with each other, although small peak is observed at 3 seconds for Run023, and the value at $100 \%$ thrust of Run023 is slightly higher than that of Run022, due to the difference of the engine operational sequence. PIF 1 increases after engine ignition both at Run022 and Run023. In the normal test of Run023, PIF1 shows a constant value while the engine is operated at $100 \%$ thrust. On the other hand, PIF1 decreases gradually until the termination of the engine at 63 seconds in the abnormal test of Run022. The SEMs with the threshold of $99.5 \%$ for the $1^{\text {st }}$ and $2^{\text {nd }}$ PCA modes are shown in Fig. 9. Same with the previous discussion in Section 5.1, Run018 is chosen as the nominal test case $\left(R_{0}\right)$ for computing the DTW distance. It is observed in the result of the $1^{\text {st }}$ PCA mode shown in Fig. 9(a) that the failure signal indicating Run022 successfully appears here and there in the SEM. Especially, most of the combinations of PIF1 indicate the failure signal, because the current system failure appears directly on PIF1. Phase plane trajectory of $1^{\text {st }}$ PCA mode between PIF1 and PCIG $\left(\mathcal{O}^{(1)}\right.$ (PCIG, PIF1) $)$ is compared in Fig. 10. Each phase plane trajectory is colored by the time from engine ignition. Under normal conditions, the phase plane trajectory is shaped as if two sticks are arranged horizontally close to each other. Whereas, those two sticks are located separately in the fault test of Run022. As shown in Fig. 11, the hierarchical clustering is applied to the degree of dissimilarity between the phase plane trajectories of the 23 firing tests in order to classify them. Note that Run022 deviates from the others, which indicates the possibility of a fault. PIDF located at the FTP is also shown in Fig. 8. Since PIDF shows the similar trend with PIF1, the combinations with PIDF should also indicate failure at Run022. However, as shown in Fig. 9(a), number of the combinations with PIDF

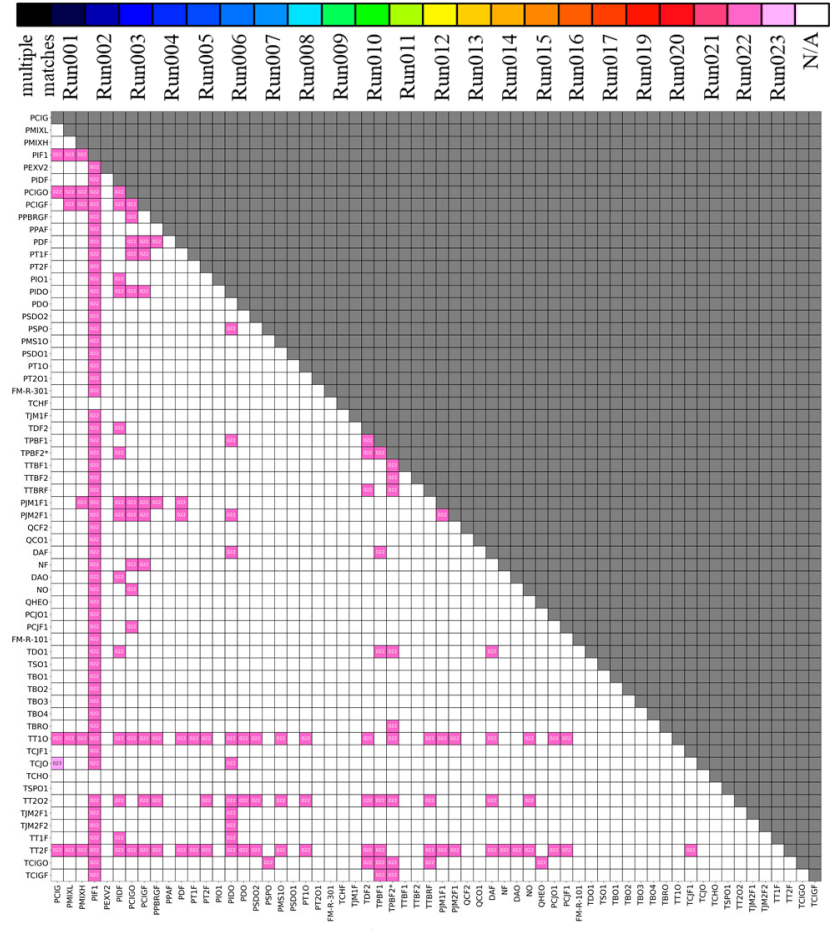

(a) $1^{\text {st }}$ PCA mode.

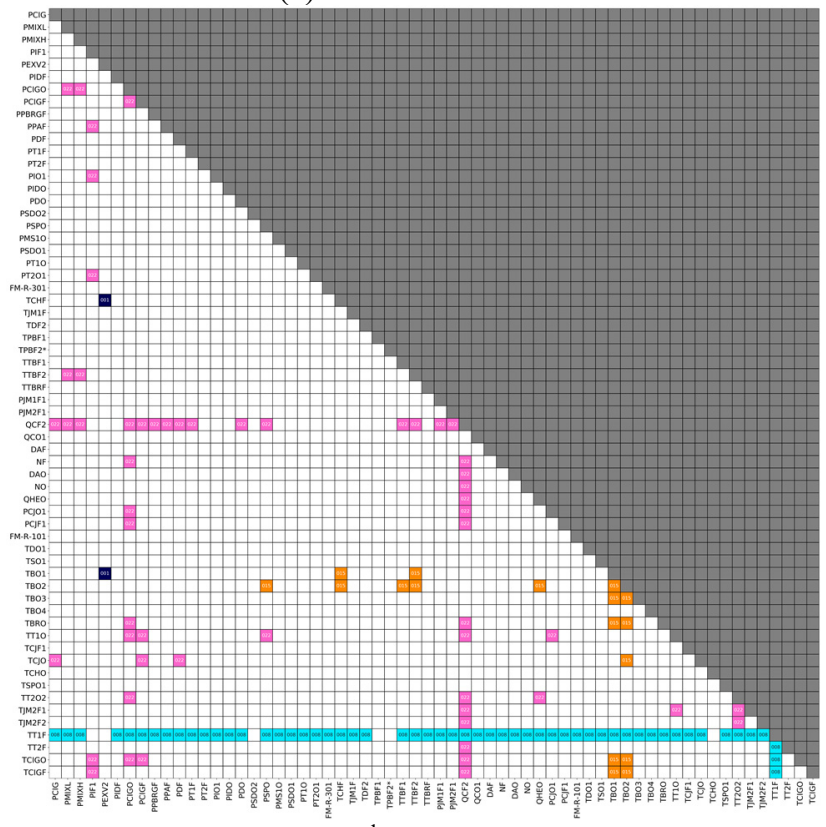

(b) $2^{\text {nd }}$ PCA mode.

Figure9. SEM with threshold for anomaly score of $99.5 \%$ for simulated system failure in FTP inducer.

indicating the failure signal of Run022 is less than that with PIF1. According to Fig. 8, PIDF at Run023 shows a peak at the time of engine shutdown. However, there is no peak at Run022. The difference observed here originates in the difference of the engine operational sequence. PIDF at four tests of Run001, Run002, Run020, and Run021 also has no peak at the time of engine shutdown. As a result, TNR is poor 
for the combinations with PIDF. The SEM for the $2^{\text {nd }}$ PCA mode is shown in Fig 9(b). Those sensor combinations showing the failure signal of Run022 is limited. Since the change in sensor value due to the system failure focused here is slow, the change does not appear in the $2^{\text {nd }}$ PCA mode. On the other hand, the sensor failure of TT1F at Run008 is correctly detected at the swath elements related to TT1F. From the above discussion, it is proved that the present bivariate time-series analysis has a capability to identify the system failure. In addition, the ability to distinguish the system and sensor failures from the SEM is demonstrated.

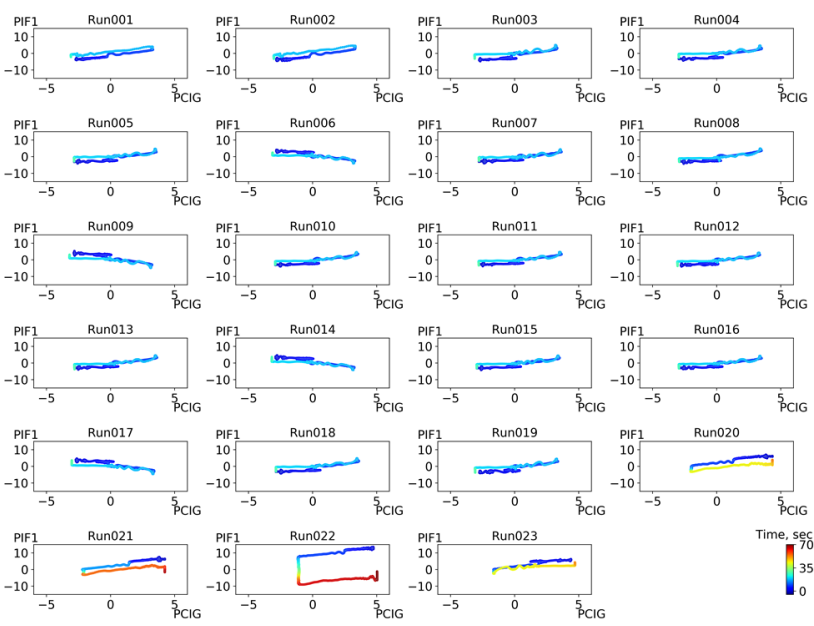

Figure10. Phase plane trajectories $\mathcal{O}_{R_{k}}^{(1)}$ (PCIG, PIF1) for 23 firing tests $(k=1, \cdots, 23)$.

\section{CONClusion}

A bivariate time-series analysis method based on the phase plane trajectory of feature vectors extracted by principal component analysis is developed for fault detection of a reusable liquid-propellant rocket engine. From the sensorstate estimation matrix showing the state of all sensor combinations, it is possible to distinguish between sensor and system failure. And because the dissimilarity of the phase plane trajectory is evaluated by dynamic time warping, this method is also robust to deviations of the timing of the engine operational sequence due to changes in control input, individual differences of the engine, and drift of the operating conditions that occur even in the same engine. Static-firing test results of the reusable rocket engine obtained at the Japan Aerospace Exploration Agency are employed for demonstration of the present method. The present method is thus able to detect thermocouple faults that occurred in one of the 19 firing tests of 62 sensors, even in the deviation of the engine operational sequence between the static-firing tests due to control input. The present method is also able to detects the system failure from 23 firing tests. Furthermore, the ability to distinguish system and sensor failure from the sensor-state estimation matrix is also demonstrated.

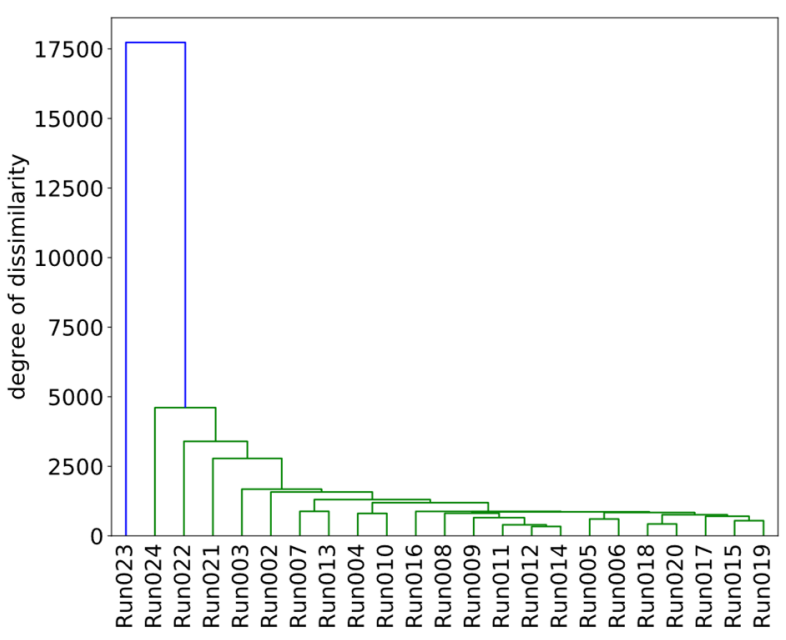

Figure11. Dendrogram of the degree of dissimilarity, $G_{\mathrm{PIF} 1, \mathrm{PCIG}}^{(1)}\left(\mathrm{Run} 018, R_{k}\right)(k=1, \cdots, 17,19, \cdots, 23)$.

\section{REFERENCES}

Bahlmann, C., and Burkhardt, H. (2004). The Writer Independent Online Handwriting Recognition System frog on hand and Cluster Generative Statistical Dynamic Time Warping, IEEE Transaction on pattern analysis and machine learning intelligence, Vol.26, No.3, pp.299-310.

Fujimaki, R., Yairi, T., and Machida, K. (2005). An approach to spacecraft anomaly detection problem using kernel feature space, Proceedings of the $11^{\text {th }}$ ACM SIGKDD international conference on knowledge discovery in data mining, pp.401-410.

Fujino, S., Mori, M., and Takeuchi, T. (1991). Performance of hyperplane ordering on vector computers, Journal of Computational and Applied Mathematics, Vol.38., pp.125136.

Hawman, M.W., Galinaitis, W.S., Tulpule, S., and Mattedi, A.K. (1990). Framework for a Space Shuttle Main Engine Health Monitoring System. NASA CR-185224.

Iannetti, A., Marzat, J., Piet-Lahanier, Ordonneau, G., and Vingert L. (2015). Fault diagnosis benchmark for a rocket engine demonstrator, Proceedings of $9^{\text {th }}$ International Federation of Automatic Control Symposium on Fault Detection, Supervision and Safety for Technical Processes SAFEPROCESS 2015, Paris, France, pp. 895-900. https://doi.org/10.1016/j.ifacol.2015.09.640

Inatani, Y., Ogawa, H., Naruo, Y., Nonaka, S. and Tokudome, S. (2010). Recent Progress Toward Reusable Sounding Rocket, 61 st International Astronautical Congress (IAC), IAC-10-D2.4.9.

Jolliffe, I.T. (2002) Principal Component Analysis, Second Edition. New York: Springer.

Kimura, T., Hashimoto, T., Sato, M., Takada, S., Moriya, S., Yagishita, T., Naruo, Y., Ogawa, H., Ito, T., Obase, K., and Ohmura, H. (2016), Reusable Rocket Engine: 
Firing Tests and Lifetime Analysis of Combustion Chamber, Journal of Propulsion and Power, Vol.32, No.5, pp. 1087-1094. https://doi.org/10.2514/1.B35973

Kurita,T., Sekita,I., and Otsu,N. (1994), Invariant Distance Measures for Planar Shapes Based on Complex Autoregressive Model, Pattern Recognition, Vol.27, No.7, pp.903-911. doi: 10.1109/TPAMI.2004.1262308

Nonaka, S., Ito, T., Ogawa, H., and Inatani, Y. (2015), Technologies and Verifications for Reusable Sounding Rocket System, 66th International Astronautical Congress (IAC), IAC-15-D2.5.4.

Sato, M., Hashimoto, T., Takada, S., Kimura, T., Naruo, Y., Yagishita,T., Ito, T., Moriya, S., Niu, K., Kaneko, T., Obase, K., and Takagi,H. (2014), Inspection in Life Critical Components and Maintainability of Reusable Sounding Rocket Engine, $65^{\text {th }}$ International Astronautical Congress (IAC), IAC-14-C4.P36.

Sato, M., Hashimoto, T., Soma, T., and Mizoguchi, T. (2017). Failure Detection of Liquid Propellant Rocket Engine Using System Invariant Analysis Technology, 68th International Astronautical Congress (IAC), IAC-17C4.1.12, 2017.

Schwabacher, M., Oza, N., and Mathews, B. (2009). Unsupervised Anomaly Detection for Liquid-Fueled Rocket Propulsion Health Monitoring, Journal of Aerospace Computing, Information, and Communication, Vol.6, pp.464-482. https://doi.org/10.2514/1.42783

$\mathrm{Wu}$, J. (2005) Liquid-propellant rocket engines healthmonitoring-a survey, Acta Astronautica, Vol.56, pp.347356. https://doi.org/10.1016/j.actaastro.2004.05.070 